\title{
ZONEAMENTO AGROCLIMÁTICO DE NOGUEIRA-MACADÂMIA PARA O BRASIL ${ }^{1}$
}

\author{
LUCAS MENDES SCHNEIDER 2 , GLAUCO DE SOUZA ROLIM ${ }^{3}$, \\ GRACIELA DA ROCHA SOBIERAJSKI ${ }^{4}$, ANGÉLICA PRELA-PANTANO ${ }^{5}$, \\ MARCOS JOSÉ PERDONÁ 6
}

RESUMO- A noz-de-macadâmia tem grande potencial de mercado, mas ainda é pouco explorada no Brasil. As condições de clima e solo de uma região têm grande influência na fenologia, qualidade, produtividade e sustentabilidade do cultivo. O objetivo deste trabalho foi realizar o zoneamento agroclimático da nogueiramacadâmia para o Brasil. Para tanto, foram utilizadas informações que relacionassem o desenvolvimento da planta e suas necessidades climáticas para estabelecimento de classes de aptidão e posterior mapeamento das regiões aptas, marginais e inaptas para o cultivo. Foram utilizados dados médios de 30 anos de temperatura do ar e precipitação mensal de 1.073 estações climatológicas no Brasil. Como resultado, observou-se que São Paulo, Rio de Janeiro, Espírito Santo, sul de Minas Gerais, leste de Mato Grosso do Sul e oeste do Paraná apresentam condições favoráveis para o cultivo de macadâmia.

Termos para indexação: noz, temperatura, chuva, Macadamia integrifolia.

\section{AGROCLIMATIC ZONING FOR MACADAMIA NUT TO BRAZIL}

\begin{abstract}
The macadamia nut has a growing market with great potential, but still little explored in Brazil. The regional climate and soil conditions have extreme importance to crop sustainability exerting great influence on phenology, quality and productivity. The aim of this study was to make the agroclimatical zoning of macadamia nut to Brazil. For this purpose, information about the relationship of climate requirements and plant development were used to establish classes of capability and subsequent mapping of suitable, marginal and unsuitable regions for nut production. Mean air temperature and precipitation data from 1073 Brazilian weather stations were used. Each station had at least 30 years of data. As a result São Paulo, Rio de Janeiro, Espírito Santo, southern Minas Gerais, eastern Mato Grosso do Sul, and west of Paraná had favorable conditions for macadamia nuts cultivation.
\end{abstract}

Index terms: nut, temperature, rain, Macadamia integrifolia.

\section{INTRODUÇÃO}

A nogueira-macadâmia é uma árvore subtropical da família Proteaceae, a qual apresenta quatro espécies, com destaque para Macadamia integrifolia e Macadamia tetraphylla (SOBIERAJSKI et al., 2006). O nome macadâmia foi dado em homenagem a John MacAdam, que caracterizou diversas espécies de plantas no continente australiano (PIMENTEL, 2007).

A Austrália é a maior produtora de noz em casca do mundo, com 35.500 toneladas, o que equivale a aproximadamente 10.000 ton de amêndoas (AMS, 2012), correspondendo a 32\% da produção mundial (HAL, 2012). O Brasil é o $6^{\circ}$ país em expor- tação, correspondendo a $2 \%$ da produção mundial. Possui uma área plantada de aproximadamente 6.000 ha, com produção anual de 3.200 toneladas de noz em casca, sendo que os principais Estados produtores são: São Paulo, Espírito Santo, Rio de Janeiro e Bahia (FRANÇA, 2007; PIMENTEL, 2007; SOBIERAJSKI et al., 2006).

A noz é utilizada para alimentação, além de ser usada para fins paisagísticos, produção de óleo, cosméticos e fármacos (DIERBERGER; MARINO NETTO, 1985; DIERBERGER et al., 1986; PIMENTEL, 2007). A maior quantidade de noz produzida é de aproximadamente $70 \mathrm{~kg}$ de nozes ano ${ }^{-1}$ planta $^{-1}$ sob condições ótimas de cultivo (DIERBERGER; MARINO NETTO, 1985), entretanto a produção

\footnotetext{
'(Trabalho 206-11). Recebido em: 08-08-2011. Aceito para publicação em: 06-03-2012. Programa de iniciação científica PIBIC-CNPq 2Estudante Engenharia Ambiental, PUC-Campinas, E-mail: lcsmschneider@hotmail.com.

${ }^{3}$ Eng. Agro, Prof. Dr., Depto. de Ciências Exatas, UNESP-Jaboticabal-SP, E-mail: rolim@fcav.unesp.br

${ }^{4}$ Eng. Agro, Dr., Pesquisadora Científico IAC, E-mail: sobierajski@iac.sp.gov.br

${ }^{5}$ Eng. Agro, Dr., Pesquisadora Científico IAC, Caixa Postal 28, 13020-970 Campinas (SP) E-mail: angelica@iac.sp.gov.br

${ }^{6}$ Eng. Agro, Pesquisador Científico APTA, Polo Ribeirão Preto. CEP 14030-670 E-mail: marcosperdona@apta.sp.gov.br
} 
comercial em média varia de 10 a $46 \mathrm{~kg}$ de nozes ano $^{-1}$ planta $^{-1}$ (ITO et al., 1983; SOBIERAJSKI et al., 2006).

O prolongado período juvenil e o elevado custo de processamento são os principais gargalos do cultivo, refletindo-se no longo período de retorno do capital investido para a formação do pomar (PIMENTEL, 2007). Entretanto, a vida útil de um pomar de macadâmia pode ultrapassar 60 anos, desde que as condições de solo e clima sejam adequadas ao cultivo (SÃO JOSÉ, 1991).

As condições de clima e solo de uma região para a implantação do cultivo são de extrema importância para o desenvolvimento, florescimento e produção (SÃO JOSÉ, 1991).

A precipitação pluviométrica ideal para o cultivo da noz-macadâmia deve ser superior a 1.000 $\mathrm{mm}$ anuais (STOREY, 1969). Apesar de tomar por base o total de precipitação ocorrida durante o ano, deve-se ressaltar que a distribuição destas durante os meses também é importante (SÃO JOSÉ, 1991), sendo que a irrigação é recomendada durante os períodos críticos, como na floração (STEPHENSON et al., 2003).

A temperatura é um dos fatores climáticos que influênciam diretamente no crescimento e na produtividade da planta. A temperatura ótima situa-se entre $20^{\circ} \mathrm{C}$ e $25^{\circ} \mathrm{C}$ (TROCHOULIAS; LAHAV, 1983). $\mathrm{Na}$ época da dormência e com as folhas retiradas, as plantas podem suportar temperaturas inferiores a $-6,5^{\circ} \mathrm{C}$ por curtos períodos. (DIERBERGER; MARINO NETTO, 1985). A altitude, relacionada com a temperatura média anual é outro fator que influencia na produtividade e na qualidade da noz. No Havaí, a nogueira tem produtividades elevadas desde o nível do mar até $750 \mathrm{~m}$ de altitude. Neste local, existem plantios até a $1.500 \mathrm{~m}$, porém o crescimento e a produção são menores, e as cascas das nozes são mais grossas (DIERBERGER; MARINO NETTO, 1985). O ideal é que as plantações estejam entre 100 e 600 $\mathrm{m}$ de altitude (HAMILTON, 1987).

$\mathrm{O}$ vento interfere fortemente no desenvolvimento da macadâmia, sendo recomendada a instalação de quebra-ventos bem planejados. Outra opção é o plantio em depressões e/ou vales (DIERBERGER; MARINO NETTO, 1985; HAMILTON; FUKUNAGA, 1959).

A fenologia da macadâmia é fortemente afetada pelas condições meteorológicas (Figura 1). O estádio fenológico que inicia o ciclo produtivo é o intumescimento das gemas, que ocorre no final de abril no Brasil (estádio 1), segundo escala fenológica proposta por SOBIERAJSKI et al., 2007.

$\mathrm{O}$ intumescimento das gemas (estádio 1) começa no final de abril, coincidindo com a queda de temperatura (SACRAMENTO; PEREIRA, 2003). Temperaturas noturnas adequadas estão entre $11^{\circ} \mathrm{C} \mathrm{e}$ $15^{\circ} \mathrm{C}$, com um período variável de dormência de 50 a 96 dias. A dormência é quebrada com o aumento da temperatura mínima e a ocorrência de chuvas (MONCUR et al., 1985)

O alongamento e o crescimento das inflorescências (estádios 2, 3,4) iniciam-se mais cedo nos locais frios, sugerindo possível requerimento de frio para o desenvolvimento floral (estádios 3;4) (MONCUR et al., 1985). O crescimento do racemo pode ter influência na produção potencial por planta através do decréscimo do número de flores devido à alta temperatura (MONCUR et al., 1985; SACRAMENTO; PEREIRA, 2003).

Shigeura e Ooka (1984) verificaram, em condições de laboratório, que a temperatura noturna afeta a emissão de racemos (estádio 2), sendo que as temperaturas ideais se situam entre $12^{\circ} \mathrm{C}$ e $18^{\circ} \mathrm{C}$. Entretanto, São José (1991) constatou uma amplitude menor de temperaturas ideais noturnas, entre $16^{\circ} \mathrm{Ce}$ $18^{\circ} \mathrm{C}$, por um período de 30 a 60 dias, para estimular o crescimento do racemo (estádio 2).

As chuvas durante a época do florescimento (estádio 5) contribuem para a polinização das flores nos racemos, determinando a quantidade de flores viáveis (estádios 6, 7, 8) (DIERBERGER; MARINO NETTO, 1985).

O crescimento dos frutos (estádios 9, 10) e o acúmulo de óleo são favorecidos em dias amenos no verão, não tolerando temperaturas extremas diárias (STEPHENSON et al., 1986A).

A demanda de água também é alta durante o verão, e qualquer estresse, provavelmente, interfere na fotossíntese e no preenchimento das nozes (STEPHENSON et al., 1986B). As chuvas durante a época do florescimento (estádio 5), contribuem para a polinização das flores nos racemos, determinando a quantidade de flores viáveis (estádios 6, 7, 8) (DIERBERGER; MARINO NETTO, 1985).

Com relação à maturação dos frutos (estádio 11), a necessidade hídrica é baixa comparada à época de floração e frutificação (SOBIERAJSKI et al., 2006).

A fenologia da macadâmia exerce grande influência na qualidade e na produtividade. $\mathrm{O}$ crescimento vegetativo interfere na qualidade das nozes, principalmente durante a frutificação efetiva (estádio 8), o rápido crescimento e o acúmulo de óleo (estádios 9,10,11). Nessa época, o crescimento vegetativo compete com o desenvolvimento das nozes pelos fotoassimilados (STEPHENSON et al., 1986B; JONES, 1939). 
Em relação à produtividade, o estádio 8 define o número de nozes por planta. $\mathrm{O}$ grande número de folhas fotossintetizantes durante os estádios $8,9,10$ e 11, formadas previamente, favorece o crescimento das nozes e a acumulação de óleo (STEPHENSON et al., 1986B).

A partir dos conhecimentos climáticos favoráveis à planta, podem-se definir regiões aptas, inaptas ou marginais para o seu cultivo. Com o uso de um sistema de informações geográficas (SIG), é possível a espacialização das informações, elaborando-se zoneamentos agroclimáticos.

Alguns exemplos de zoneamento agroclimático para o cultivo de macadâmia foram encontrados para as condições brasileiras. Por exemplo, Ciiagro (2012), baseado no trabalho de IAC (1977), determinou o zoneamento para fruteiras subtropicais, dentre elas a macadâmia, para o Estado de São Paulo. EPAGRI (2012) determinou o zoneamento agroclimático para a macadâmia em Santa Catarina. A EMBRAPA (2003) realizou o zoneamento agroecológico, que leva em conta, além das condições climáticas, as condições de fertilidade dos solos, para o Estado do Rio de Janeiro. E Franco et al. (1991) que realizaram o zoneamento agroclimático para macadâmia no sudeste da Bahia.

O objetivo deste trabalho foi elaborar o zoneamento agroclimático da cultura de nogueiramacadâmia para o Brasil.

\section{MATERIAL E MÉTODOS}

Os dados utilizados para a elaboração do zoneamento agroclimático da cultura de macadâmia foram retirados de 1.073 estações meteorológicas no Brasil. Foram utilizados dados mensais médios de, no mínimo, 30 anos (normalmente entre 1961 a 2000), de estações do Instituto Agronômico (IAC), Superintendência do Desenvolvimento do Nordeste (SUDENE), Instituto Nacional de Meteorologia (INMET), Instituto Agronômico do Paraná (IAPAR), Departamento Nacional de Obras Contra as Secas (DNOCS) e Departamento Nacional de Meteorologia (DNMET) (Figura 2).

As variáveis climáticas utilizadas foram definidas pelas necessidades do cultivo da macadâmia: Precipitação total anual (P), Precipitação no inverno (Pi), temperatura média anual $(\mathrm{T})$ e temperatura no inverno (Ti), como sugerido por São José (1991) e Trochoulias e Lahav (1983). Para o inverno, foram considerados os meses de junho, julho e agosto.

A caracterização das regiões aptas, marginais e inaptas para o cultivo da macadâmia foi determi- nada combinando as diferentes variáveis climáticas, de acordo com diferentes autores. Segundo Storey (1969), adaptado neste trabalho para condições brasileiras, as regiões aptas climaticamente para o cultivo são aquelas com total de Precipitação anual (P) entre $1.000 \mathrm{~mm} \leq \mathrm{P}<2.300 \mathrm{~mm}$. Foram consideradas regiões marginais quando $800 \mathrm{~mm} \leq \mathrm{P}<$ $1.000 \mathrm{~mm}$ e $\mathrm{P} \geq 2.300 \mathrm{~mm}$ e inaptas devido à baixa precipitação quando $\mathrm{P}<800 \mathrm{~mm}$.

Segundo São José (1991), além do total de precipitação anual, é importante considerar a distribuição das chuvas durante o ciclo do cultivo. Nos dados utilizados para o trabalho, observou-se que, em importantes regiões produtoras de São Paulo, Bahia e Minas Gerais, a precipitação no inverno (Pi) mínima é ao redor de $40 \mathrm{~mm}$. Dessa forma, considerou-se que os locais aptos apresentam total de chuva de inverno $(\mathrm{Pi}) \geq 40 \mathrm{~mm}$ e marginais com $\mathrm{Pi}<40 \mathrm{~mm}$.

Trochoulias e Lahav (1983) determinaram a faixa ótima de valores da temperatura média anual (T) para a noz-macadâmia. Neste trabalho, considerou-se que os valores ótimos estão entre $19^{\circ} \mathrm{C}$ $\leq \mathrm{T}<24^{\circ} \mathrm{C}$, determinando assim as regiões aptas. Consideraram-se também mais quatro condições para o desenvolvimento da nogueira: Inapto devido à elevada temperatura $\mathrm{T} \geq 27^{\circ} \mathrm{C}$; marginal pela elevada temperatura $24{ }^{\circ} \mathrm{C} \leq \mathrm{T}<27^{\circ} \mathrm{C}$; marginal devido à baixa temperatura $17^{\circ} \mathrm{C} \leq \mathrm{T}<19^{\circ} \mathrm{C}$, e inapto quando $\mathrm{T}<17^{\circ} \mathrm{C}$.

Considerou-se também a temperatura no inverno (Ti) em que as regiões aptas necessitam de uma temperatura $\mathrm{Ti} \leq 21^{\circ} \mathrm{C}$, a qual favorece o crescimento do racemo; em caso de $\mathrm{Ti}>21^{\circ} \mathrm{C}$, as regiões foram consideradas marginais.

Para a determinação das regiões aptas, marginais e inaptas, foi necessária a combinação de $\mathrm{P}, \mathrm{Pi}$, $\mathrm{T}$ e Ti (Tabela 1). Uma região foi considerada apta quando todas as condições foram aptas. Já para os locais inaptos bastava que uma condição estivesse dentro da classe inapta. Os locais marginais são aqueles em que a região não foi considerada inapta, mais apresentou restrições quanto à precipitação e/ ou temperatura.

Com a utilização do sistema de informação geográfica ArcGIS 9.0 (C) ESRI, foi feita a interpolação espacial dos valores de $\mathrm{P}, \mathrm{Pi}$, T e Ti para todas as localidades brasileiras a partir do método de krigagem (KRIGE, 1951), utilizando o modelo esférico com um vizinho e uma resolução de $0,25^{\circ}$, obtendo-se, assim, os mapas P, Pi, T e Ti nas classes propostas (Tabela 1). Com a interpolação dos quatro mapas, segundo critérios já mencionados, foi obtido o zoneamento agroclimático da cultura de macadâmia para o Brasil. 


\section{RESULTADOS E DISCUSSÃO}

O Brasil apresenta alta precipitação anual; sendo assim, encontram-se grandes regiões com precipitações apropriadas para o cultivo da noz no país (Figura 3).

As regiões litorâneas de quase todo o Brasil dispõem de chuvas suficientes para suprir as necessidades hídricas da nogueira. Apenas no litoral do Rio Grande do Norte e Ceará, a precipitação anual é baixa (Figura 3.A). Este resultado está de acordo com o trabalho desenvolvido por Franco et al. (1991), no qual os autores mencionaram que o litoral da Bahia tem precipitação ideal para o plantio da macadâmia.

Os Estados onde a média da precipitação de inverno (Figura 3.B) não é o suficiente para uma boa produção de macadâmia são: Minas Gerais, Goiás, Tocantins, parte do Maranhão, Piauí e oeste da Bahia, sendo necessária, em muitos anos, a suplementação por irrigação. As regiões Sul e Norte do Brasil apresentam chuva no inverno suficiente para um bom desenvolvimento da nogueira. Os Estados de São Paulo, Rio de Janeiro, Espírito Santo, Mato Grosso do Sul, Sergipe e Alagoas apresentam condições de precipitação anual e de inverno adequadas à produção do cultivo.

A serra catarinense e a serra gaúcha, devido às altitudes maiores que $750 \mathrm{~m}$, apresentam-se inaptas para o cultivo devido ao potencial de diminuição do crescimento e da produção, decorrentes das baixas temperaturas (Figura 3.C). Nessas condições, as cascas das nozes serão mais grossas, dificultando o beneficiamento (DIERBERGER; MARINO NETTO, 1985). Ainda ao Sul do País, encontram-se também regiões adequadas ou com restrições, como a região de fronteira com a Argentina. As regiões Norte, Nordeste e parte do Centro-Oeste do Brasil apresentam restrições ao cultivo devido às temperaturas elevadas destes locais. Entretanto, apenas uma pequena região entre os Estados de Piauí e Maranhão foi considerada inadequada para a cultura devido às altas temperaturas.

$\mathrm{O}$ mapa relacionado à temperatura no inverno (Figura 3.D) apresenta as regiões Sul por inteiro e grande parte da região Sudeste (poucas localidades nos Estados de São Paulo e Minas Gerais onde as temperaturas no inverno são superiores a $21^{\circ} \mathrm{C}$ ) como regiões apropriadas para o cultivo. Os Estados do Mato Grosso do Sul, Goiás e Bahia ainda apresentam ao sul de suas extensões, localidades aptas para o cultivo. Existe também pequena parte ao sul de Mato Grosso com temperaturas no inverno apropriadas.
AMS (2012) afirma que árvores jovens de macadâmia podem ser severamente afetadas por geadas com temperaturas até $-1^{\circ} \mathrm{C} \mathrm{a}-2^{\circ} \mathrm{C}$; já as árvores maduras suportam curtos períodos com temperaturas até $-6^{\circ} \mathrm{C}$. Salienta-se que, neste estudo em escala macroclimática, não foram levados em consideração eventos meteorológicos adversos em escala topoclimática como as geadas, que possuem alta frequência de ocorrência no Rio Grande do Sul, Santa Catarina, regiões de São Paulo e sul de Minas Gerais (ROLIM; CAMARGO, 2010).

As regiões Norte, Nordeste e partes do Centro-Oeste e Sudeste foram consideradas marginais para o cultivo devido às temperaturas de inverno, pois não apresentam as temperaturas ideais que favorecem o crescimento do racemo. Como resultado final, o zoneamento da noz-macadâmia (Figura 4) demonstra que São Paulo, Rio de Janeiro, Espírito Santo, leste de Mato Grosso do Sul, oeste do Paraná e litoral de Santa Catarina apresentam áreas com clima favorável para o sucesso das plantações. Grande parte da área central do Brasil apresenta condições marginais para o cultivo, e o nordeste apresenta áreas inaptas devido à baixa precipitação. Esses resultados estão semelhantes aos do Ciiagro (2012), que indicou que grande parte do Estado de São Paulo é apto para o cultivo, aos da e Embrapa (2003), para o Rio de Janeiro. Nota-se, ainda, que apesar de o trabalho da EPAGRI (2012) para Santa Catarina ter levado em consideração a ocorrência de geadas para o zoneamento, o presente trabalho obteve resultados semelhantes a partir dos critérios utilizados. Para ambos os zoneamentos, as regiões do Alto Vale do Rio Itajaí (chamadas de zona agroecológica 2A) e Carbonífera, Extremo sul e Colonial serrana (zona agroecológica $2 \mathrm{~B}$ ) foram classificadas inaptas para o cultivo da macadâmia.

Embora o cultivo ainda apresente pequena área de produção, como, São Paulo, o zoneamento indica grandes áreas climáticas potenciais para expansão desse cultivo no Brasil, como sul de Minas Gerais, Mato Grosso do Sul e oeste do Paraná.

As variáveis climáticas utilizadas foram definidas pelas necessidades do cultivo da macadâmia: Precipitação total anual (P), Precipitação no inverno (Pi) temperatura média anual $(\mathrm{T})$ e temperatura no inverno (Ti), como sugerido por São José (1991) e Trochoulias e Lahav (1983). Para o inverno, foram considerados os meses de junho, julho e agosto. 


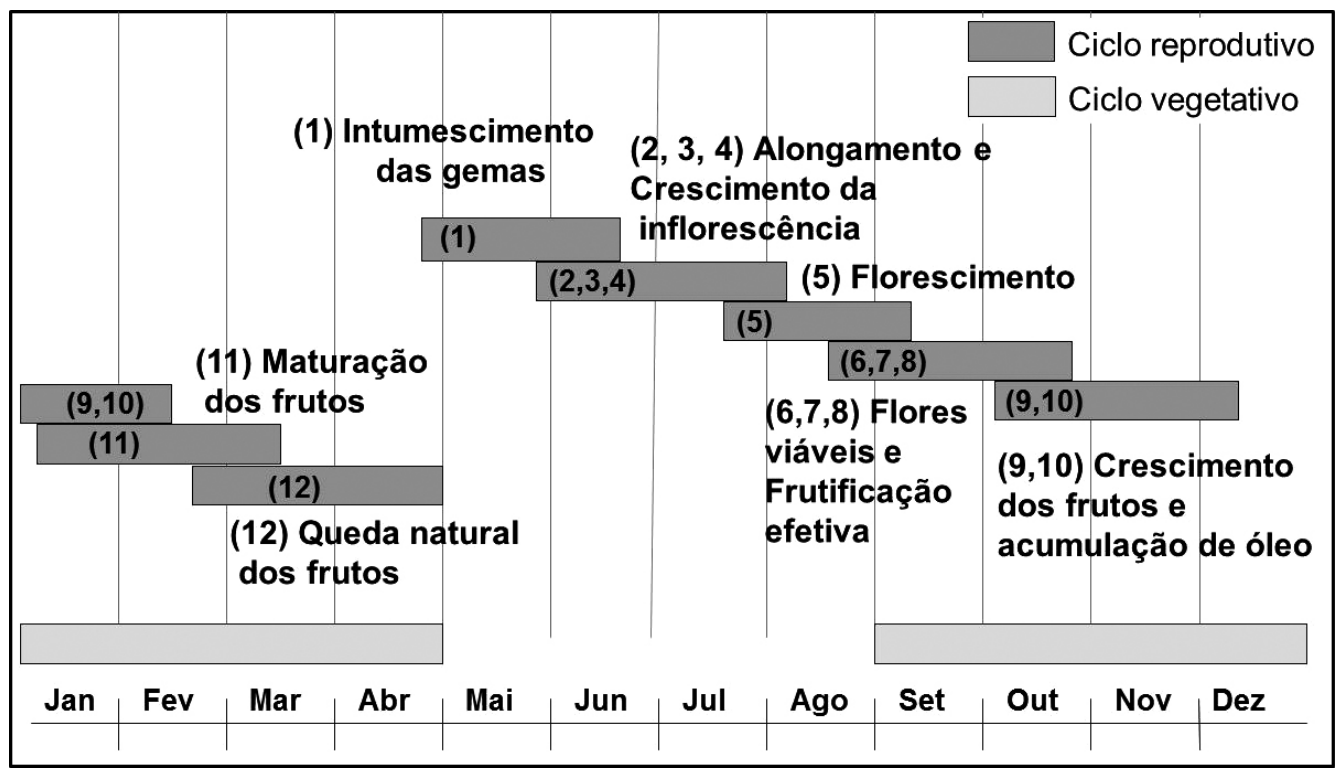

FIGURA 1- Duração dos subperíodos do desenvolvimento da macadâmia em condições brasileiras e estádios fenológicos: (1) Gemas intumescidas, primeiros indícios de diferenciação das gemas florais; (2) Crescimento do racemo, período em que ocorre a elongação do racemo floral; (3) formação dos botões florais; (4) flores em pré-antese; (5) flores abertas; (6) flores senescentes; (7) flores fecundadas; (8) frutificação efetiva; (9) crescimento dos frutos, início do acúmulo de óleo; (10) final do período de crescimento dos frutos; (11) frutos maduros, alteração de coloração e abertura do carpelo; (12) queda natural dos frutos (baseado em escala fenológica proposta por Sobierajski et al., 2007).

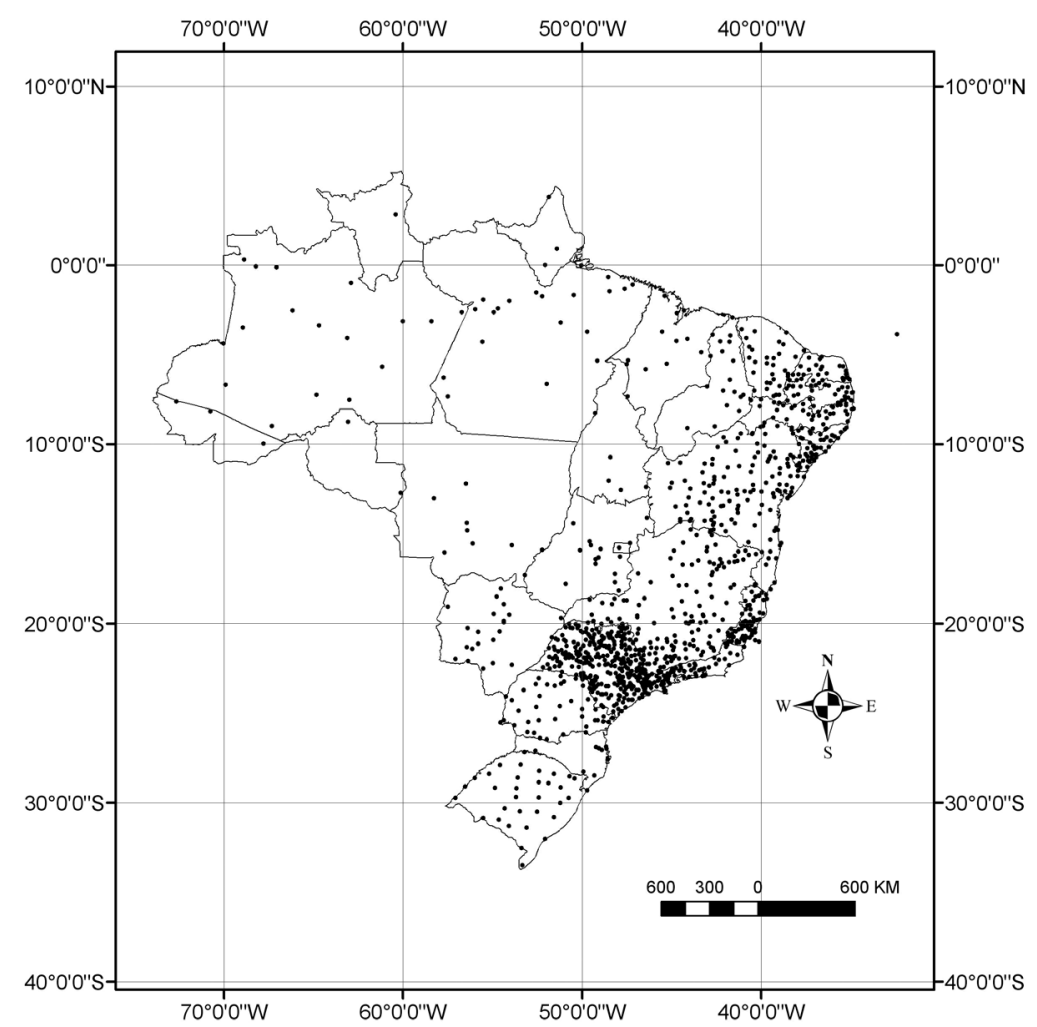

FIGURA 2- Estações meteorológicas utilizadas no trabalho. 

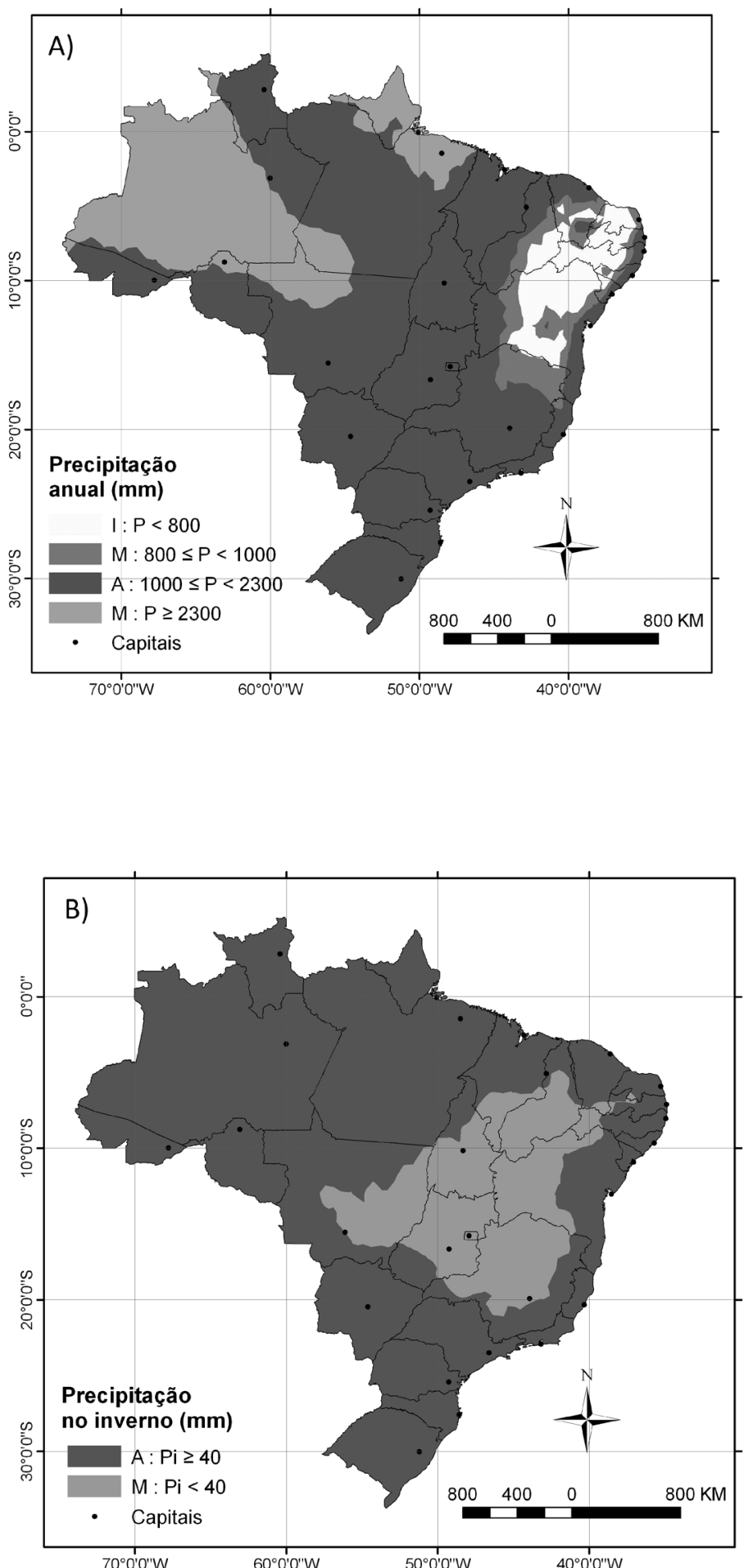

continua... 

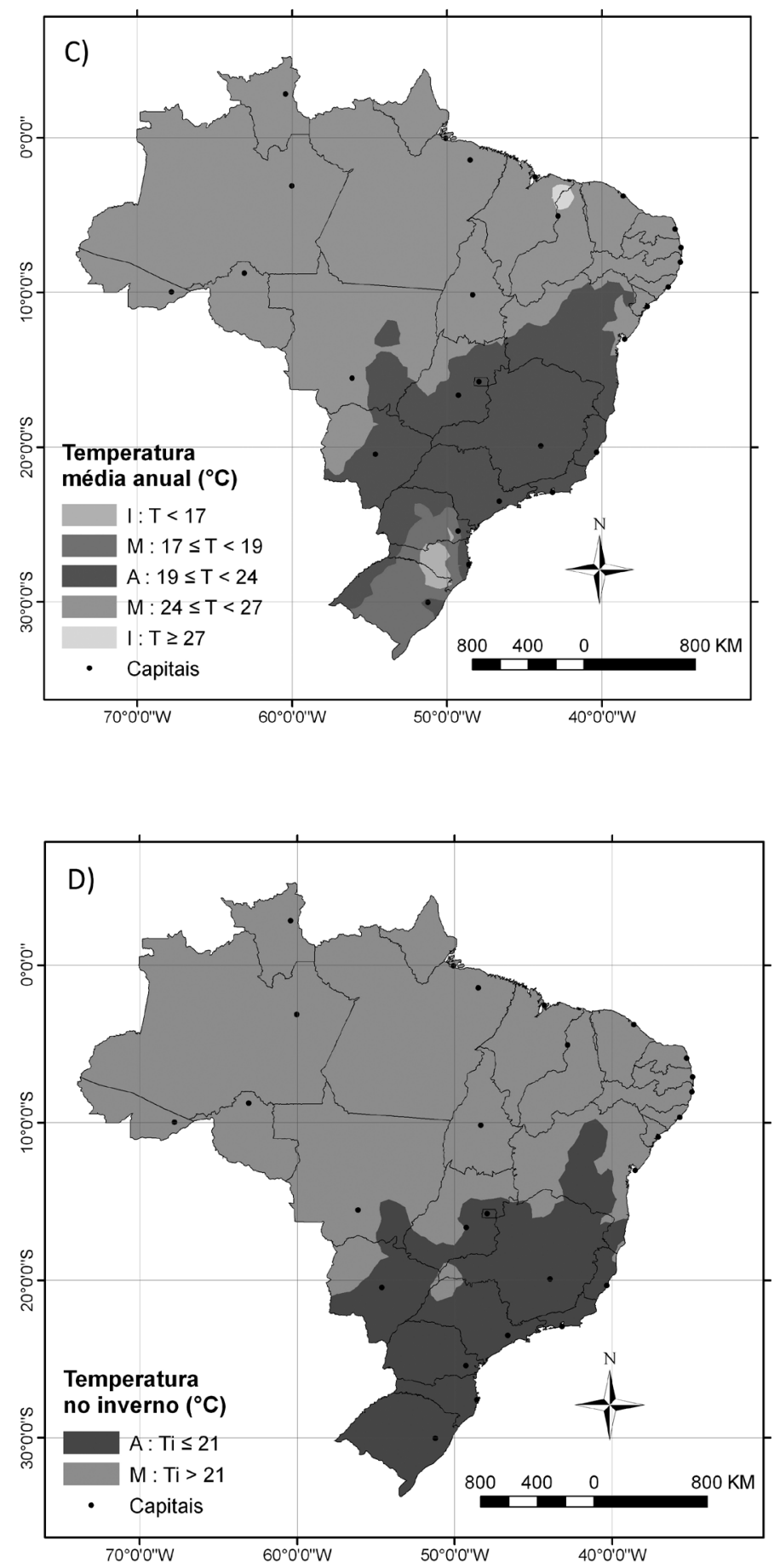

FIGURA 3- Mapa de aptidão com relação à: A) Precipitação anual (P) (mm); B) Precipitação no inverno (Pi) (junho, julho e agosto); C) Temperatura média anual (T) $\left({ }^{\circ} \mathrm{C}\right)$; D) Temperatura no inverno (Ti), para o cultivo da nogueira-macadâmia no Brasil. "A" indica apto, "M" marginal e "I" inapto para o cultivo. 


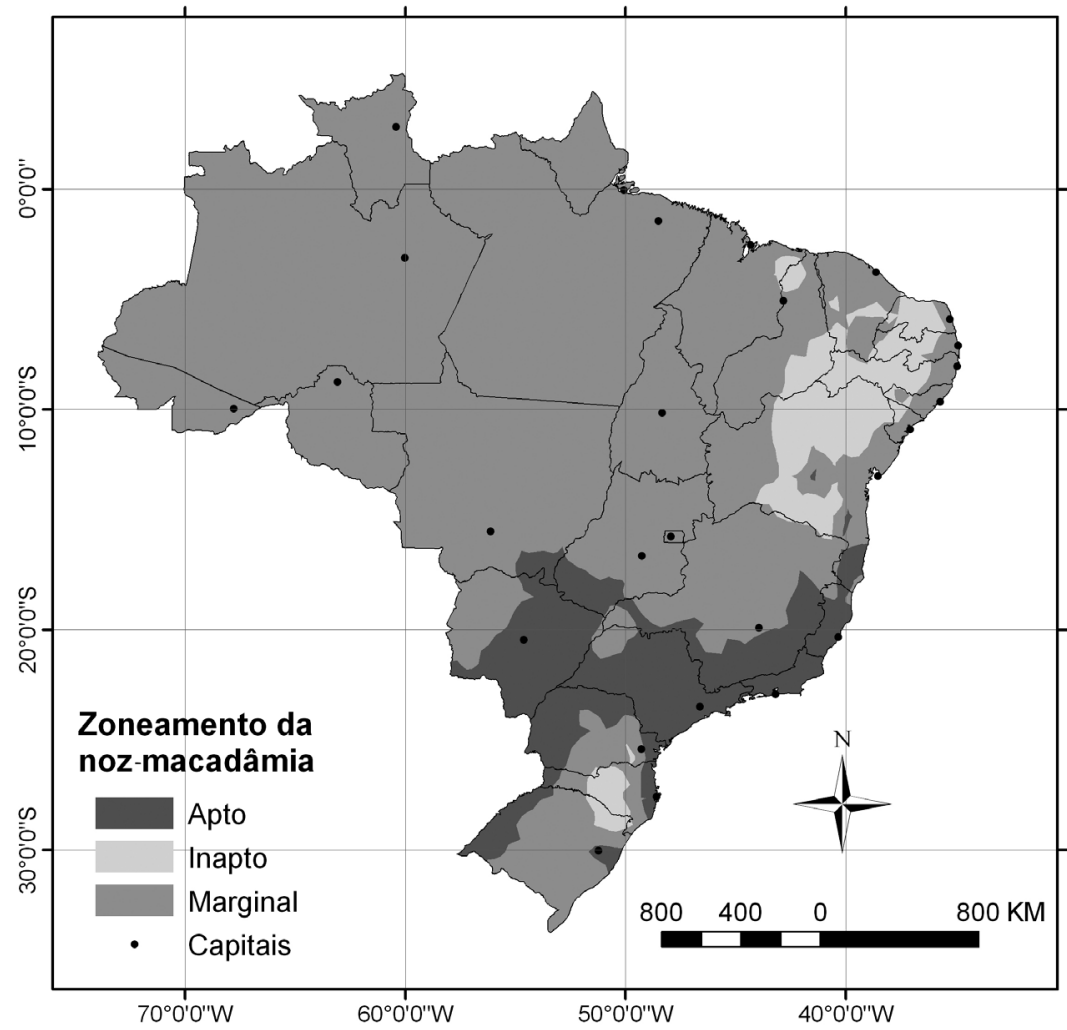

FIGURA 4- Zoneamento agroclimático para o cultivo de nogueira-macadâmia para o Brasil.

TABELA 1- Definição das variáveis climáticas para a determinação das classes de aptidão para o cultivo da nogueira-macadâmia.

\begin{tabular}{|c|c|c|c|}
\hline \multicolumn{2}{|c|}{ Precipitação anual (P) (mm) } & \multicolumn{2}{|c|}{ Precipitação no inverno $(\mathrm{Pi})(\mathrm{mm})$} \\
\hline $\mathrm{P} \geq 2300$ & - Marginal & $\mathrm{Pi} \geq 40$ & - Apto \\
\hline $1000 \leq \mathrm{P}<2300$ & - Apto & $\mathrm{Pi}<40$ & - Marginal \\
\hline $800 \leq \mathrm{P}<1000$ & - Marginal & & \\
\hline $\mathrm{P}<800$ & - Inapto & & \\
\hline \multicolumn{2}{|c|}{ Temperatura média anual $(\mathrm{T})\left({ }^{\circ} \mathrm{C}\right)$} & \multicolumn{2}{|c|}{ Temperatura no inverno $(\mathrm{Ti})\left({ }^{\circ} \mathrm{C}\right)$} \\
\hline $\mathrm{T} \geq 27$ & - Inapto & $\mathrm{Ti}>21$ & - Marginal \\
\hline $24 \leq \mathrm{T}<27$ & - Marginal & $\mathrm{Ti} \leq 21$ & - Apto \\
\hline $19 \leq \mathrm{T}<24$ & - Apto & & \\
\hline $17 \leq \mathrm{T}<19$ & - Marginal & & \\
\hline $\mathrm{T}<17$ & - Inapto & & \\
\hline
\end{tabular}




\section{CONCLUSÕES}

O zoneamento agroclimático permite a classificação das regiões por aptidão climática e demonstra que grande parte de São Paulo, Rio de Janeiro, Espírito Santo, sul de Minas Gerais, leste do Mato Grosso do Sul e oeste do Paraná apresentam disponibilidade climática favorável ao cultivo da nogueira.

\section{AGRADECIMENTOS}

Agradecemos o apoio dos Engenheiros Agrônomos Dr. Pedro Luís Blasi de Toledo Piza e Leonardo Massaharu Moriya, da empresa QueenNut Macadâmia, do município de Dois Córregos- SP, que pelas discussões técnicas contribuíram sobremaneira com o trabalho.

\section{REFERÊNCIAS}

AMS - Australian Macadamia Society. Frequentlyasked questions, Macadamias in the Orchard, What growing conditions best suit macadamias?. Lismore, 2012. Disponível em: <macadamias.org/ pages/faqs $>$. Acesso em: 13 fev. 2012.

CIIAGRO - Centro Integrado de Informações Agrometeorológicas. Aptidão climática das frutas de clima subtropical. Campinas,, 2012. Disponível em: $<$ www.ciiagro.sp.gov.br/zoneamento/ frutassubtropical.htm>. Acesso em: 13 fev. 2012.

DIERBERGER, J.E. A noz macadamia no Brasil. Limeira: Ed. Dierberger Indústria e Comércio, 1986. 8p.

DieRBERGER, J.E.; NETTO, L.M. Noz macadâmia: uma nova opção para a fruticultura brasileira. São Paulo: Nobel, 1985. p.45-66.

EMBRAPA - Empresa Brasileira de Pesquisa Agropecuária. Zoneamento agroecológico do estado do Rio de Janeiro. Rio de Janeiro: Embrapa Solos, 2003. 143p. (Boletim de Pesquisa e Desenvolvimento, 33)

EPAGRI-CIRAM -Empresa de Pesquisa Agropecuária e Extensão Rural de Santa Catarina, Centro de Informações de Recursos Ambientais e de Hidrometeorologia de Santa Catarina. Zoneamento agroecológico e socieconômico. Florianópolis, 2012. Disponível em: http://www.ciram.epagri.sc.gov.br/ ciram_arquivos/portal/agricultura/zoneAgroecologico/ZonAgroeco.pdf>. Acesso em: 13 fev. 2012.
FRANÇA, B.H.C. Dossiê técnico: macadâmia, cultivo e produtos derivados. Rio de Janeiro: REDETEC, 2007. 21p.

FRANCO, M.A.G.; SACRAMENTO, C.K.; PEREIRA, M.A. Aptidão climática para o cultivo da macadâmia no sudeste da Bahia. Macadâmia: tecnologia de produção e comercialização. Vitória da Conquista: DFZ/UESB, 1991. p.29-38.

HAL, Horticulture Australia Limited. Macadamia industry annual report 2010/2011. Disponível em: $<$ cms2live.horticulture.com.au/admin/assest/library/ annual reports/pdfs/PDF File 183.pdf $>$. Acesso em: 13 fev. 2012.

HAMILTON, R.A.; FUKUNAGA, E.T. Growing macadamia nuts in Hawaii. Hawaii: Agricultural Experiment Station of University of Hawaii, 1959. 51p. (Bulletin, 121)

HAMILTON, R.A.; ITO, P.J; FOSS, S. Testing adaptation of Hawaiian macadamia varieties at high elevations. Yearbook California Macadamia Society, Fallbrook, v.27, p.53-59, 1987.

IAC - Instituto Agronômico. Atlas do Zoneamento agrícola do estado de São Paulo. Campinas: Secretaria da Agricultura, 1977. 100p.

ITO, P.J.; HAMILTON, R.A.; HIRAE, H.H. Yield and quality of five major Macadamia cultivars in Hawaii. Tropical Agriculture, Surrey, v.60, p 6465,1983

JONES, W.W. A study of developmental changes in composition of the macadamia. Plant Physiology, Minneapolis, v.14, p.755-768, 1939.

KRIGE, D. A statistical approach to some basic mine valuation problems on the witwatersrand. Journal of Chemical, Metal and Mining Society of South Africa, v. 52, p.119-139. 1951.

MONCUR, M.W.; STEPHENSON, R.A.; TROCHOULIAS, T. Floral development of Macadamia integrifolia Maiden \& Betche under Australian conditions. Scientia Horticulturae, Amsterdam, v.27, p.87-96. 1985.

PIMENTEL, L.D. A cultura da macadâmia. Revista Brasileira de Fruticultura, Jaboticabal, v.29, n.3, p.414-716, 2007. 
ROLIM, G.S.; CAMARGO, M.B.P. Geada. In: MATTHES, L.A.F.; UZZO, R.P. Palmeiras ornamentais: produção e cultivo. Campinas: Ed. Fundag, 2010. p.173-187.

SACRAMENTO, C.K.; PEREIRA, F.M. Fenologia da floração da nogueira-macadâmia (Macadamia integrifolia Maiden \& Betche)nas condições climáticas de Jaboticabal, São Paulo, Brasil. Revista Brasileira de Fruticultura, Jaboticabal, v.25, n.1, p.19-22, 2003.

SACRAMENTO, C.K.A. Macadamicultura no Brasil In: SAO JOSE, A.R. (Ed.). Macadâmia: tecnologia de produção e comercialização. Vitória da Conquista: DFZ-UESB, 1991. p.192-197. (não consta no texto)

SÃO JOSÉ, A.R. Exigências edafoclimáticas para a cultura da macadâmia. In: São José, A. R. (Org.). Macadâmia: tecnologia de produção e comercialização. Vitória da Conquista: DFZ/UESB, 1991. p.29-38.

SHIGEURA, G.T.; OOKA, H. Macadamia nuts in Hawaii: history and production. Hawaii: College of Tropical Agriculture and Human Resources, 1984. p.91 (Res. Ext. Series, 039).

SMITH, L.S. New species and notes on Queensland plants. Proceedings of the Royal Society of Queensland.v. 67, p. 39-40, 1956.

SOBIERAJSKI, G.R.; BARBOSA, W.; BETTIOL NETO, J.E.; CHAGAS, E.A.; CAMPODALL'ORTO, F.A. Caracterização dos estágios fenológicos em sete cultivares e seleções de nogueiramacadâmia. Revista Brasileira de Fruticultura. Jaboticabal, v.29, n.3, p.690-694, 2007.
SOBIERAJSKI, G.R.; FRANCISCO, V.L.F.S.; ROCHA, P.; GHILARDI, A.A.; MAIA, M.L. Nozmacadâmia: produção, mercado e situação no Estado de São Paulo. Informações Econômicas, São Paulo, v.36, n.5, p.25-36, 2006.

STEPHENSON, R.A.; CULL, B.W.; MAYER, D.G. Effects of site, climate, cultivar, flushing, and soil and leaf nutrient status on yields of macadamia in South East Queensland, Scientia Horticulturae, Amsterdam, v.30, p.227-235, 1986b.

STEPHENSON, R.A.; CULL, B.W.; STOCK, J. Vegetative flushing patterns of macadamia trees in south east Queensland. Scientia Horticulturae, Amsterdam, v.30, p.53-63, 1986a.

STEPHENSON, R.A.; GALLAGHER, E.C.; DOOGAN, V.J. Macadamia responses to mild water stress at different phenological stages. Australian Journal of Agricultural Research, Victoria, v.54, p.67-75, 2003.

STOREY, W.B. Macadamia. In: HALEVY, A.H. CRC handbook of flowering III. Boca Raton: CRC Press, 1969

TROCHOULIAS, T.; LAHAV, E. The effect of temperature on growth and dry matter production of macadamia. Scientia Horticulturae, Amsterdam, v.19, p.167-176, 1983 